\title{
Radiologic evaluation of nonalcoholic fatty liver Disease
}

\author{
Ahmed EL-Eraky ${ }^{1, *}$, Hatem Elalfy ${ }^{1}$, Talal Amer ${ }^{2}$, Raghda Farag ${ }^{1}$, Mona Arafa ${ }^{1}$ \\ ${ }^{1}$ Tropical Medicine Department, Mansoura University, Mansoura, Egypt; ${ }^{2}$ Diagnostic and Interventional Radiology \\ Department, Faculty of Medicine, Mansoura University, Mansoura, Egypt.
}

\begin{abstract}
Nonalcoholic Fatty Liver Disease (NAFLD) is a clinical syndrome characterized by predominant macrovesicular steatosis of the liver. NAFLD comprises a range of liver conditions varying in severity of hepatocytes injury and resulting fibrosis-cirrhosis risk. Among these, hepatic steatosis (fatty liver) is referred to as NAFL, and nonalcoholic fatty liver (NAFL) is defined as a more grave process with both fat and inflammation in the liver that over time can cause liver cirrhosis (steatohepatitis). Liver biopsy is the gold standard method to differentiate, whether the patient with fatty liver has only steatosis, or NASH. Unfortunately, liver biopsy has well-known limitations (invasiveness and sampling variability) and cannot be proposed for all patients, especially given the high prevalence of NAFLD worldwide. This review discuss the radiologic evaluation of liver steatosis and fibrosis for patients with NAFLD.
\end{abstract}

\section{Introduction}

Non-alcoholic fatty liver disease ( NAFLD) is the leading cause of liver disease worldwide and is estimated to affect $25 \%$ of the global population ${ }^{1}$. The histological definition of NAFLD is the presence of triacylglycerol (TAG) droplets in $>5 \%$ of hepatocytes, in the absence of excessive alcohol consumption or the use of steatogenic drugs ${ }^{2}$. Histologically, NAFLD ranges in severity from steatosis alone (NAFL) to steatohepatitis (NASH), where steatosis is associated with hepatocellular injury, inflammation and fibrosis. Approximately $40 \%$ of patients with NAFLD will develop progressive fibrosis, which can result in cirrhosis ${ }^{3,4}$.

Liver biopsy is currently considered the gold standard of diagnosis of NASH, however, it is invasive and limited by cost and sampling error ${ }^{5}$. In addition ,both patients and clinicians are often hesitant to pursue biopsy due to its invasive nature with potential for clinical complications including severe bleeding and rarely death ${ }^{6}$. In real-world clinical practice, providers often use a combination of noninvasive serum tests, imaging results and endoscopic

Keywords:

Received: 15-10-2021 ; Accepted: 7-11-2021

* Corresponding author. Email: omtyomty2@yahoo.com findings to arrive at a personalized diagnosis and risk stratification for an individual patient ${ }^{7}$.

Radiologic Imaging of NAFLD

The clinical importance of NAFLD and the limitations of liver biopsy have increased the need for accurate and noninvasive imaging methods to evaluate NAFLD. To date, various imaging methods have been utilized to evaluate patients with NAFLD summarized in (Table 1). Each imaging method for liver fat quantification has its own advantages and disadvantages as shown in (Table 2). More recently, several imaging methods that measure liver stiffness have been investigated for their usefulness in assessing inflammation and fibrosis in patients with NAFLD ${ }^{8}$.

Table 1: Summary of Radiologic tools for evaluation of NAFLD

Radiologic tools for evaluation of NAFLD

Conventional Ultrasonography (CUS)

Doppler Ultrasonography (DUS)

Computed Tomography (CT)

Magnetic Resonance Imaging Proton Density Fat Fraction( MRI-PDFF)

Controlled Attenuation Parameter (CAP)

Vibration controlled Transient Elastography (VCTE)

Acoustic Radiation Force Impulse (ARFI)

Shear Wave Elastography (SWE)

Magnetic Resonance Elastography (MRE)

Table 2: Commonly used modalities for liver fat quantification ${ }^{8}$

\begin{tabular}{|c|c|c|c|c|c|}
\hline Modality & Cost & Accuracy & $\begin{array}{l}\text { Point } \\
\text { of } \\
\text { care }\end{array}$ & Quantitative & Caveats \\
\hline CUS & + & ++ & Yes & No & $\begin{array}{l}\text { May fail in obesity and } \\
\text { in ironoverload and cirrhosis }\end{array}$ \\
\hline CAP & + & ++ & Yes & $\begin{array}{l}\text { Yes, but not } \\
\text { linear in higher } \\
\text { liver fat } \\
\text { content }\end{array}$ & $\begin{array}{l}\text { Affected by type of } \\
\text { probe and fibrosis }\end{array}$ \\
\hline CT & ++ & ++ & No & $\begin{array}{l}\text { Semi- } \\
\text { quantitative }\end{array}$ & Ionizing radiation \\
\hline $\begin{array}{l}\text { MRI- } \\
\text { PDFF }\end{array}$ & ++ & +++ & No & Yes & Not suitable for screening \\
\hline
\end{tabular}




\section{Ultrasound (US) for evaluating hepatic steatosis.}

Hepatic steatosis on US appears as a diffuse increase in hepatic echogenicity, or "bright liver", due to increased reflection of US from the liver parenchyma, which is caused by intracellular accumulation of fat vacuoles. US evaluation of hepatic steatosis typically consists of a qualitative visual assessment of hepatic echogenicity, measurements of the difference between the liver and kidneys (Figure 1) in echo amplitude, evaluation of echo penetration into the deep portion of the liver, and determination of the clarity of blood vessel structures in the liver. Severity is usually graded clinically using a fourpoint scale, as follows: normal (grade 0), mild (grade 1), moderate (grade 2), and severe (grade 3) ${ }^{9}$. In patients without coexisting liver disease, US offers a fairly accurate diagnosis of moderate-to-severe hepatic steatosis (i.e., defined as histologic degree $\geq 30 \%$ or $33 \%$ ), with reported sensitivity ranging from $81.8 \%$ to $100.0 \%$ and specificity as high as $98 \%$. In contrast, US was not accurate in diagnosing hepatic steatosis when all degrees of steatosis were considered (i.e., $\geq 3 \%$ or $5 \%$ ), with a reported sensitivity ranging from $53.3 \%$ to $66.6 \%$ and specificity ranging from $77.0 \%$ to $93.1 \%{ }^{10}$. As hepatic fibrosis may also increase hepatic echogenicity, the presence of underlying chronic liver disease may reduce the accuracy of US in the diagnosis of hepatic steatosis. For example, one study that included hepatitis $\mathrm{C}$ patients found that US had a sensitivity of $60 \%$ and a specificity of $73 \%$ in detecting histologically proven moderate-to-severe hepatic steatosis ${ }^{11}$. One major limitation of US is the substantial intra- and inter-observer variability. Another limitation of US is the qualitative nature of the current four-point grading system. Although this grading system is the most widely used for US evaluation of hepatic steatosis in practice, it is too simplistic to account for small alterations in steatosis severity on follow-up. Thus, US may be inadequate for evaluating patients with NAFLD after therapeutic intervention. To overcome the limitations of US, computer-assisted quantitative US techniques were developed for the assessment of hepatic steatosis. These techniques employ dedicated post-processing software programs to analyze US echo amplitude, attenuation, and/or texture-based information. The most robust parameter is the computerized hepatorenal index, defined as the ratio of the echo intensities of the liver and renal cortex. The results of two related studies were very promising, with this index demonstrating sensitivities of $92.7 \%$ and $100 \%$ and specificities of $91 \%$ and $92.5 \%$ in diagnosing hepatic steatosis $\geq 5 \%{ }^{12}$. Computerized quantitative analysis methods for US may be able to overcome these limitations, but they require further clinical validation. A closely related, but a non-imaging technique is controlled attenuation parameter (CAP). Of these, CAP is the most validated and commercially available through Echosens (Paris, France), the manufacturer of FibroScan. CAP is an objective measure of ultrasound attenuation and can be performed using the appropriately selected FibroScan probe to measure liver stiffness and steatosis simultaneously ${ }^{13}$. A preliminary study in a mixed population of diffuse liver disease reported high correlation of CAP with histological steatosis grade (correlation coefficient 0.81 ) with excellent severity grading performance and high reproducibility ${ }^{14}$. In a NAFLD population, validation data are still incomplete, but CAP has thus far shown promise as a standardized quantitative US biomarker for steatosis ${ }^{15}$. However, further validation is needed, including evaluation of newer probes optimized for obese patients.

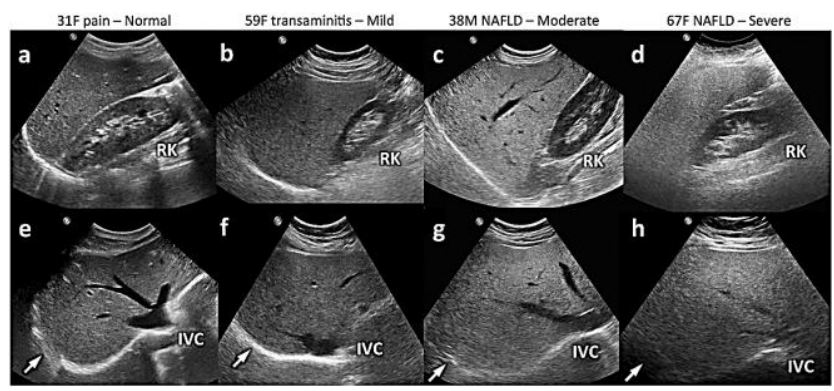

Figure 1. Ultrasound of NAFLD examples. In normal liver, the liver parenchyma is slightly more echogenic (i.e., brighter) than the right kidney (a). Posterior structures are well seen, including diaphragm (e). In the steatotic liver, the parenchyma becomes increasingly more echogenic than the kidney (b-d) and deep structures, including the diaphragm (arrow), which become progres-sively blurred $(\mathrm{f}-\mathrm{h})^{16}$.

\section{Computerized CT for evaluating hepatic steatosis.}

CT evaluation of hepatic steatosis is based on the attenuation values of the liver parenchyma, evaluated as Hounsfield units (HUs), and dependent on tissue composition. As the attenuation value of fat (i.e., approximately $-100 \mathrm{HU}$ ) is much lower than that of soft tissue, hepatic steatosis lowers the attenuation of liver parenchyma ${ }^{17}$. unenhanced CT scans are usually preferred to avoid the potential errors in contrast-enhanced CT caused by variations in liver attenuation related to contrast injection methods and scan timing. Several quantitative $\mathrm{CT}$ indices have been used to assess hepatic steatosis, with the two most frequently used being the absolute liver attenuation value (i.e., HU liver) and the liver-to-spleen difference in attenuation (i.e., CTL-S) (Figure 2). Despite $\mathrm{HU}$ liver showing a stronger correlation with histologic degree of hepatic steatosis than CTL-S, HU liver may be subject to errors resulting from variations in attenuation values across CT scanners from different vendors. This error can be avoided, however, by using CTL-S, which incorporates spleen attenuation as an internal control ${ }^{18}$. CT was quite accurate for the diagnosis of moderate-tosevere steatosis but was not as accurate for detecting mild steatosis ${ }^{9}$. To establish a more generalized threshold value of $\mathrm{CT}$ indices for the diagnosis of hepatic steatosis, a normal reference range for CTL-S (1-18 HU) was established using histologically proven, nonsteatotic 
healthy livers. An HU liver of 48 and a CTL-S of -2 were found to be threshold values for a $100 \%$ specific diagnosis of moderate-to-severe hepatic steatosis. Several factors other than hepatic fat can influence liver attenuation on $\mathrm{CT}$, including the presence of excess iron in the liver and the ingestion of certain drugs such as amiodarone ${ }^{19}$. Dualenergy CT can differentiate among several chemical components in tissue, by using $\mathrm{X}$ rays at two different energy levels. To date, studies revealed that its use did not improve the accuracy of conventional single-energy CT in assessing hepatic steatosis ${ }^{20}$. The low accuracy of CT in detecting a mild degree of hepatic steatosis suggests that, this method may not be suitable for the evaluation of NAFLD because patients with NAFLD frequently have a mild degree of steatosis ${ }^{21}$. Moreover, the potential hazard of ionizing radiation makes CT unsuitable for use in children or for longitudinal monitoring of patients with NAFLD. CT for longitudinal follow-up of hepatic steatosis is also uncertain, due to a lack of knowledge about the reproducibility of serial $\mathrm{CT}$ measurements and the assay sensitivity of CT indices in detecting small changes in the severity of hepatic steatosis. Therefore, CT may not be appropriate for the evaluation of NAFLD, although it may be useful in evaluating hepatic steatosis in specific clinical scenarios. For example, CT can be used successfully to detect moderate-to-severe hepatic steatosis in donor candidates for liver transplantation ${ }^{19}$, and CT measurement of fat in the liver may be useful for patients at risk of metabolic syndrome ${ }^{22}$.
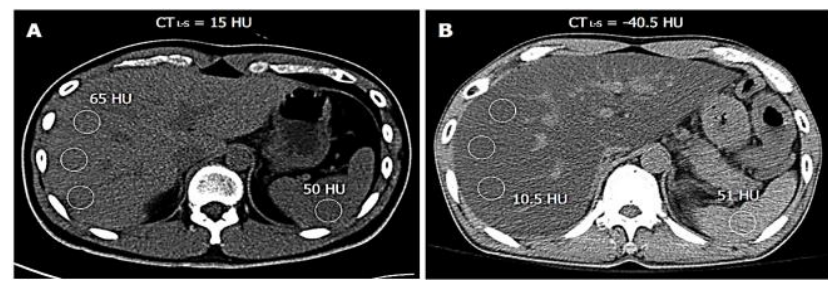

Figure 2. CT evaluation of hepatic steatosis using CTL-S index. A: CT image of a normal liver, showing that its attenuation $(65 \mathrm{HU})$ measured using regions-of-interest (white circles) was higher than that of the spleen $(50 \mathrm{HU})$, and the CTL-S value was $15 \mathrm{HU}$, which lies within the normal reference range; B: CT image of a steatotic liver, showing hepatic attenuation (10.5 HU) much lower than that of the spleen $(51 \mathrm{HU})$, making the CTL-S value -40.5 $\mathrm{HU}$, far below the normal reference range and indicating moderate-to-severe hepatic steatosis ${ }^{23}$.

Magnetic resonance imaging (MRI) methods for evaluating hepatic steatosis.

Unlike CT and US, which evaluate hepatic steatosis through proxy parameters (echogenicity and attenuation, respectively), MRI and magnetic resonance spectroscopy (MRS) can more directly measure the quantity of hepatic fat in an objective manner using the quantitative index proton density fat fraction (PDFF), defined as the amount of protons bound to fat divided by the amount of all

protons in the liver, including those bound to fat and water. The basic magnetic resonance (MR) physics used in both techniques to differentiate protons in fat from those in water is the chemical-shift phenomenon, i.e., the difference in MR frequency between the protons in fat and water ${ }^{23}$. Several MR imaging techniques are available, but by far the most widely used in clinical practice is called in-phase (IP) and opposed-phase (OP) imaging, also known as chemical shift imaging or two-point Dixon technique ${ }^{24}$. This dual-echo CSI technique is universally available on all modern clinical 1.5 and 3 Tesla (T) systems and included in most clinical abdominal MR examinations ${ }^{16}$. Multi parametric MRI refers to use of multiple quantitative (parametric) MRI features or measures with several possibilities for combinations 25 . Currently, multi-parametric quantitative MRI offers the most comprehensive set of NAFLD biomarkers for clinical care and research, not only allowing objective quantification of fat, but also iron and fibrosis, in a single examination i.e., a virtual liver biopsy ${ }^{16}$. Studies consistently demonstrated that MRS and MRI outperform CT and US in the diagnosis and grading of hepatic steatosis, even when MRS and MRI were performed without any of the sophisticated corrective methods e.g, (correction of $\mathrm{T} 2$ or $\mathrm{T} 2 *$ effects) ${ }^{9},{ }^{10}$. The MRI sensitivities and specificities in detecting histologic steatosis $\geq 5 \%$ were $76.7 \%-90.0 \%$ and $87.1 \%-91 \%$, respectively, and the corresponding MRS performances were $80.0 \%-91.0 \%$ and $80.2 \%-87.0 \%$, respectively 9,10 . The standard deviation of PDFF values over repeated
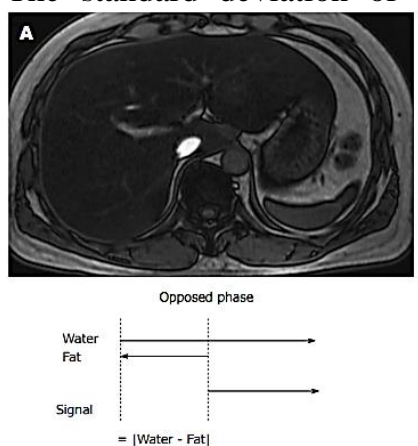

measurement was less than $1 \%$ for both MRS and MRI ${ }^{26}$

Figure 3. Dual-echo opposed-phase and in-phase chemical shift images of steatotic liver A: At opposed-phase (OP) (echo time $=2.3 \mathrm{~ms}$ at $1.5 \mathrm{~T}$ ), the protons in water and those in methylene (the largest fat moiety) are placed in opposite directions, so that the signals of these two components cancel each other. Therefore, the liver appears dark (i.e. , decreased signal); B: At in-phase (IP), the protons in water and those in methylene are positioned in the same direction so that their signals are added. Liver fat fraction can be calculated based on signal intensities on OP and IP images as (signal at IP - signal at OP) $\div 2 \times$ signal on IP; the signal fat fraction calculated with dualecho chemical shift images was not corrected for the T2* effect, and therefore may not accurately determine proton density fat fraction ${ }^{23}$. 


\section{Imaging diagnosis of NASH and elastography.}

In general, no imaging examinations have been found to accurately diagnose NASH, making liver biopsy the only reliable method of distinguishing NASH from simple steatosis. US elastography and MR elastography, however, are emerging as promising methods to diagnose NASH. US elastography and MR elastography evaluate liver stiffness by measuring the velocity of shear wave using US (US elastography) or MRI (MR elastography). Several US elastography techniques have been described, which differ in methods of shear wave generation and/or detection, including transient elastography, acoustic radiation force impulse elastography, supersonic shear wave elastography, and real-time tissue elastography (Table 3) ${ }^{27}$. These techniques were first applied to the evaluation of liver fibrosis in patients with chronic viral hepatitis, and their clinical application has recently been expanded to other liver diseases, including NAFLD. US elastography techniques have demonstrated very promising results for the diagnosis of liver fibrosis in NAFLD. They have shown a stepwise increase in liver stiffness as the severity of histologic liver fibrosis increased, and have been highly accurate in differentiating advanced liver fibrosis from mild liver fibrosis, with sensitivities ranging from $88.9 \%$ to $100 \%$ and specificities ranging from $75.0 \%$ to $100 \%$. Liver stiffness value did not correlate with the degree of hepatic steatosis or with hepatic inflammation, indicating that US elastography can assess hepatic fibrosis associated with steatosis without confounding by steatosis but would not be able to assess hepatic inflammation ${ }^{28}$. A study of MR elastography in 58 patients with NAFLD showed that liver stiffness in patients with steatosis and lobular inflammation was significantly higher than in patients with steatosis only, and significantly lower than in patients with steatosis and fibrosis ${ }^{29}$. Taken together, these results indicate that US elastography or MR elastography may play a potential role in screening for NASH and/or advanced fibrosis in patients with NAFLD ${ }^{23}$.

Table 2: Comparison between elastographic modalities.

\begin{tabular}{|c|c|c|c|c|c|}
\hline Modality & Cost & Accuracy & $\begin{array}{l}\text { Point } \\
\text { of } \\
\text { care }\end{array}$ & $\begin{array}{l}\text { Quality } \\
\text { Criteria }\end{array}$ & Caveats \\
\hline VCTE & + & ++ & Yes & Standardized & $\begin{array}{l}\text { Increased } \\
\text { variability in } \\
\text { morbid obesity and } \\
\text { cirrhosis }\end{array}$ \\
\hline ARFI/SWE & + & ++ & $\begin{array}{l}\text { Can } \\
\text { be }\end{array}$ & $\begin{array}{l}\text { QIBA* is } \\
\text { working on } \\
\text { it }\end{array}$ & $\begin{array}{l}\text { Increased } \\
\text { variability in } \\
\text { morbid obesity and } \\
\text { cirrhosis }\end{array}$ \\
\hline MRE & ++ & +++ & No & $\begin{array}{l}\text { QIBA* is } \\
\text { working on } \\
\text { it }\end{array}$ & $\begin{array}{l}\text { Excellent accuracy } \\
\text { in obesity and } \\
\text { cirrhosis May fail } \\
\text { in the setting of } \\
\text { Iron overload }\end{array}$ \\
\hline
\end{tabular}

VCTE, vibration controlled transient elastography; ARFI, acoustic radiation forced impulse imaging; SWE, shear wave elastography; MRE, magnetic resonance elastography; QIBA, Quantitative Imaging Biomarkers Alliance.

\section{Utility of Doppler US in NAFLD}

Duplex Doppler ultrasonography (US) has been found to be an important diagnostic technique in the noninvasive evaluation of hepatic vasculature and some hepatic parenchymal diseases ${ }^{30,31}$. New findings suggest that diffuse fatty infiltration of liver can alter the hemodynamics in the hepatic veins (as detected by Doppler US) as well as the hepatic artery resistance index (HARI) ${ }^{32,33}$. Magalotti et al. showed that patients with NASH have decreased portal blood flow velocity, increased intrahepatic arterial resistance, and abnormalities in the Doppler wave forms of the hepatic veins ${ }^{34}$. Additionally, some studies reported that assessment of indices of hepatic vasculature detected by Duplex Doppler improved the diagnostic performance of ultrasonography 35, 36. As a methodology, it involves no radiation exposure, readily available, inexpensive, quantitative and rapid, prolongs the duration of a typical US examination by only 1-2 minutes ${ }^{37}$

\section{A. Portal venous pulsatility index in NAFLD.}

Intra-abdominal adipose tissues can be sub-divided into intraperitoneal and retroperitoneal adipose tissues. Such regional adiposity is believed to be important because venous drainage of the intraperitoneal adipose tissue goes directly to the liver, through the portal vein, whereas the retroperitoneal adipose tissue drains into the systemic circulation. Thus, free fatty acids, glycerol, and other adipocytokines that are released from the intraperitoneal adipose tissue may influence the hepatic metabolism of glucose, triglycerides, insulin, and other substrates and hormones. The portal fat hypothesis is based on this unique pattern of venous drainage ${ }^{38}$. Portal vein pulsatility is an imaging biomarker measured by duplex Doppler assessment of the portal vein and quantified as the venous pulsatility index (VPI). VPI is calculated as (Vmax Vmin) / Vmax, where Vmax is the maximum and Vmin is the minimum pulsed-wave Doppler ultrasound-estimated velocity of blood in the portal vein. In a rabbit model of steatosis, moderate fatty liver infiltration has been shown to cause significant reductions in portal and total hepatic blood flow and microcirculation, along with significant increases in hepatic artery flow and portal pressure ${ }^{39}$. Although a few studies have investigated the distribution of VPI in patients with NAFLD, the accuracy of this
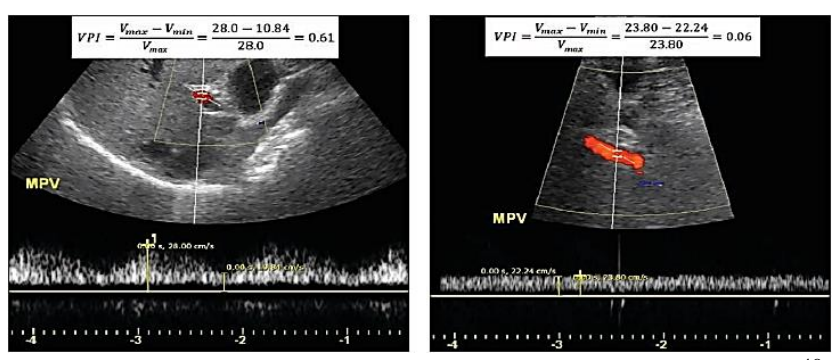

method for identifying high-risk NAFLD is not known ${ }^{40}$. Portal vein pulse Doppler values may be useful for disease diagnosis and the monitoring of responses to treatment. Improvement in these values was observed after treatment 
based on dietary modifications, increased physical activity, and administration of metformin ${ }^{34}$.

Figure 4: Calculation of portal venous pulsatility index (VPI).A, 47-year-old man with NAFLD with fibrosis stage of F0. B-mode sonographic image at level of main portal vein (MPV) with superimposed color Doppler and spectral Doppler ROIs shows how maximum (Vmax) and minimum (Vmin) velocity are calculated from spectral waveform. Calculated VPI of 0.61 is elevated and would be unlikely to reflect NAFLD. B, 59-year-old man with NAFLD with fibrosis stage of F4. Spectral Doppler waveform measured in MPV has minimal temporal variation. Low calculated VPI of 0.06 corresponds to highrisk NAFLD ${ }^{40}$.

B. Hepatic veins doppler in NAFLD.

The hepatic veins (HV) in a healthy subject have characteristic triphasic waveform pattern, which consists of three peaks; antegrade systolic and diastolic flow, and a short retrograde flow by right atrial systole ${ }^{32}$. This flow pattern is influenced by the pressure in the right atrium, the compliance of the hepatic parenchyma, and modification of the intrathoracic and intraabdominal pressures produced by respiration ${ }^{41}$. It has been demonstrated that decreased phasicity of hepatic veins with biphasic or monophasic waveform is associated with cirrhosis, fibrosis, hepatitis, transplant rejection, hepatic vein thrombosis (Budd-Chiari syndrome), hepatic veno-occlusive disease, and fatty liver 31.

HV Doppler waveform has been studied well in chronic parenchymal liver diseases in the past decade, whereas the relation of fatty liver and abnormal HV Doppler waveform was searched in only few studies, mostly as a small subgroup of patients for comparison with parenchymal liver diseases ${ }^{42}$. Although arterial blood flow increases in fatty liver, portal venous flow decreases. Blood flow changes in fatty liver might be due to hypertrophied hepatocytes that cause hv compression and subsequently decrease venous blood flow phasicity, leading to a change in hv waveforms. Adding Doppler examination to the routine conventional US examination in patients with fatty liver can provide contributive information about the severity of fat accumulation and its effect on liver perfusion ${ }^{43}$. Many research studies had inferred that diffuse fatty infiltration of the liver may cause altered flow patterns in the hepatic veins and doppler indices of hepatic artery ${ }^{42}$.

\section{Hepatic Artery Resistive Index.}

A number of studies published before the advent of Transient Elastography (TE) into clinical practice demonstrated that the assessment of some haemodynamic parameters provided by Doppler US investigation of hepatic vessels might indirectly reflect histological alterations, namely liver fibrosis. In particular, the resistance index in the hepatic and in the splenic artery (HARI and SARI, respectively) was demonstrated to increase in cirrhosis in comparison to chronic viral hepatitis ${ }^{44}$. The hepatic artery resistive index (HARI ) is used for follow-up of microcirculatory resistance in fatty liver, adult alcoholic liver disease, chronic hepatitis, and post-transplant liver patients ${ }^{45,46}$. A study conducted by Hizli et al revealed that ALT, TG, and TC levels and HARI level of obese subjects with fatty liver are significantly higher than those of lean subjects. Elevation in HARI level was also correlated significantly with BMI increase. They recommend that HARI be a preliminary candidate for the detection of early derangement of hepatic arterial perfusion due to insulin resistance ${ }^{47}$. Another Italian study found a significant inverse correlation between HARI and severity of diffuse fatty liver disease in NAFLD patients, with a significant decrease in HARI as severity of fatty disease increases ${ }^{36}$. These results substantially confirm the data published previously ${ }^{33,48}$. Additionaly, the measurement of HARI has demonstrated a significant positive correlation with fibrosis degree, as measured with NAFLD fibrosis score, suggesting that the fibrous tissue accumulation may result in increased arterial rigidity and, therefore, in a rise of resistance to flow, and that the different tissue composition of the liver (adipose versus fibrous) can influence HARI differently ${ }^{36,49}$. Gray US index (echogenicity of the liver) might not change in some of the patients who responded to therapy, or it might tend to decrease more slowly than RI index. This shows the fact that assessing the improvement in subjects with fatty liver is more reliable using Doppler US and HARI and points attention to the importance of early diagnosis and the urgent need of the characterization of hepatic vessel flow abnormalities in the NAFLD population ${ }^{50}$.

\section{Conclusion}

Radiologic tools for evaluation of NAFLD provide a promising noninvasive methods for assessment of liver steatosis and fibrosis. Providers can use a combination of noninvasive serum tests, imaging results and endoscopic findings to arrive at a personalized diagnosis and risk stratification avoiding unnecessary liver biopsies.

\section{References}

1. Younossi ZM, Koenig AB, Abdelatif D., et al. (2016). Global epidemiology of nonalcoholic fatty liver disease-Meta-analytic assessment of prevalence, incidence, and outcomes. Hepatology, 64(1):73-84.

2. Hardy T, Oakley F, Anstee QM., et al. (2016). Nonalcoholic fatty liver disease: pathogenesis and disease spectrum. Annual Review of Pathology: Mechanisms of Disease,(11):451-96.

3. Anstee QM, Targher G, Day CP., et al. (2013). Progression of NAFLD to diabetes mellitus, cardiovascular disease or cirrhosis. Nature Reviews Gastroenterology and Hepatology,10(6):330.

4. McPherson S, Hardy T, Henderson E, et al. (2015). Evidence of NAFLD progression from steatosis to fibrosing-steatohepatitis using paired biopsies: implications for prognosis and clinical management. Journal of hepatology, 62(5):1148-55. 
5. Sumida Y, Nakajima A, Itoh Y., et al. (2014). Limitations of liver biopsy and non-invasive diagnostic tests for the diagnosis of nonalcoholic fatty liver disease/nonalcoholic steatohepatitis. World J Gastroenterol, 20(2):475-85.

6. Rowe IA, and Parker R., (2015). Liver biopsy for the selection of patients with nonalcoholic steatohepatitis for clinical trials. Gastroenterology,148(1):262.

7. Tincopa MA (2020). Diagnostic and interventional circulating biomarkers in nonalcoholic steatohepatitis. Endocrinol Diabetes Metab, 3(4): 00177.

8. Loomba R. (2018). Role of imaging-based biomarkers in NAFLD: Recent advances in clinical application and future research directions. $J$ Hepatol, 68(2):296-304.

9. Lee SS, Park SH, Kim HJ, et al. (2010). Noninvasive assessment of hepatic steatosis: prospective comparison of the accuracy of imaging examinations. J Hepatol, 52(4):579-85.

10. Van Werven JR, Marsman HA, Nederveen AJ., et al. (2010). Assessment of hepatic steatosis in patients undergoing liver resection: comparison of US, CT, T1-weighted dual-echo MR imaging, and point-resolved 1H MR spectroscopy. Radiology, 256(1):159-68.

11. Hepburn MJ, Vos JA, Fillman EP, et al. (2005). The accuracy of the report of hepatic steatosis on ultrasonography in patients infected with hepatitis $\mathrm{C}$ in a clinical setting: a retrospective observational study. BMC Gastroenterol,5(1):14.

12. de Almeida e Borges VF, Diniz ALD, Cotrim HP, et al. (2013). Sonographic hepatorenal ratio: a noninvasive method to diagnose nonalcoholic steatosis. J Clin Ultrasound, 41(1):18-25.

13. Sasso M, Beaugrand M, De Ledinghen V., et al. (2010). Controlled attenuation parameter (CAP): a novel VCTE ${ }^{\mathrm{TM}}$ guided ultrasonic attenuation measurement for the evaluation of hepatic steatosis: preliminary study and validation in a cohort of patients with chronic liver disease from various causes. Ultrasound Med Biol, 36(11):1825-35.

14. Sasso M, Miette V, Sandrin L, et al (2012). The controlled attenuation parameter (CAP): a novel tool for the non-invasive evaluation of steatosis using Fibroscan®. Clin Res Hepatol Gastroenterol, 36(1):13-20.

15. de Lédinghen, V., Vergniol, J., Capdepont, M., et al. (2014). Controlled attenuation parameter (CAP) for the diagnosis of steatosis: a prospective study of 5323 examinations. Journal of hepatology, 60(5), 1026-1031.

16. Dündar, İ., and Yavuz, A. (2021). Effect of Ramadan Fasting on Liver Steatosis and Liver Volume Measured by Magnetic Resonance Imaging with Liver Function Tests and Lipid Profile. Journal of Nutrition, Fasting and Health.
17. Kim, D. Y., Park, S. H., Lee, S. S., et al. (2010). Contrast-enhanced computed tomography for the diagnosis of fatty liver: prospective study with same-day biopsy used as the reference standard. European radiology, 20(2), 359-366.

18. Pickhardt, P. J., Park, S. H., Hahn, L., et al. (2012). Specificity of unenhanced CT for non-invasive diagnosis of hepatic steatosis: implications for the investigation of the natural history of incidental steatosis. European radiology, 22(5), 1075-1082.

19. Park, Y. S., Park, S. H., Lee, S. S., et al. (2011). Biopsy-proven nonsteatotic liver in adults: estimation of reference range for difference in attenuation between the liver and the spleen at nonenhanced CT. Radiology, 258(3), 760-766.

20. Artz, N. S., Hines, C. D., Brunner, S. T., et al. (2012). Quantification of hepatic steatosis with dualenergy computed tomography: comparison with tissue reference standards and quantitative magnetic resonance imaging in the ob/ob mouse. Investigative radiology, 47(10), 603.

21. Adams, L. A., Sanderson, S., Lindor, K. D., et al. (2005). The histological course of nonalcoholic fatty liver disease: a longitudinal study of 103 patients with sequential liver biopsies. Journal of hepatology, 42(1), 132-138.

22. Ross, R. (2003). Advances in the application of imaging methods in applied and clinical physiology. Acta diabetologica, 40(1), s45-s50.

23. Lee, S. S., and Park, S. H. (2014). Radiologic evaluation of nonalcoholic fatty liver disease. World journal of gastroenterology: WJG, 20(23), 7392.

24. Merkle, E. M., and Nelson, R. C. (2006). Dual gradient-echo in-phase and opposed-phase hepatic MR imaging: a useful tool for evaluating more than fatty infiltration or fatty sparing. Radiographics, 26(5), 1409-1418.

25. Allen, A. M., Shah, V. H., Therneau, T. M., et al. (2020). Multiparametric magnetic resonance elastography improves the detection of NASH regression following bariatric surgery. Hepatology communications, 4(2), 185-192.

26. Yokoo, T., Shiehmorteza, M., Hamilton, G., et al. (2011). Estimation of hepatic proton-density fat fraction by using MR imaging at 3.0 T. Radiology, 258(3), 749-759.

27. Ochi, H., Hirooka, M., Koizumi, Y., et al. (2012). Real-time tissue elastography for evaluation of hepatic fibrosis and portal hypertension in nonalcoholic fatty liver diseases. Hepatology, 56(4), 1271-1278.

28. Wong, V. W. S., Vergniol, J., Wong, G. L. H., et al. (2010). Diagnosis of fibrosis and cirrhosis using liver stiffness measurement in nonalcoholic fatty liver disease. Hepatology, 51(2), 454-462.

29. Chen, J., Talwalkar, J. A., Yin, M., et al. (2011). Early detection of nonalcoholic steatohepatitis in patients with nonalcoholic fatty liver disease by 
using MR elastography. Radiology, 259(3), 749756.

30. Bolondi, L., Li Bassi, S., Gaiani, S., et al. (1991). Liver cirrhosis: changes of Doppler waveform of hepatic veins. Radiology, 178(2), 513-516.

31. von Herbay, A., Frieling, T., and Häussinger, D. (2001). Association between duplex Doppler sonographic flow pattern in right hepatic vein and various liver diseases. Journal of clinical ultrasound, 29(1), 25-30.

32. Oguzkurt, L., Yildirim, T., Torun, D., et al. (2005). Hepatic vein Doppler waveform in patients with diffuse fatty infiltration of the liver. European journal of radiology, 54(2), 253-257.

33. Mihmanli, I., Kantarci, F., Yilmaz, M. H., et al. (2005). Effect of diffuse fatty infiltration of the liver on hepatic artery resistance index. Journal of clinical ultrasound, 33(3), 95-99.

34. Magalotti, D., Marchesini, G., Ramilli, S., et al. (2004). Splanchnic haemodynamics in non-alcoholic fatty liver disease: effect of a dietary/pharmacological treatment: a pilot study. Digestive and liver disease, 36(6), 406-411.

35. Balci, A., Karazincir, S., Sumbas, H., et al. (2008). Effects of diffuse fatty infiltration of the liver on portal vein flow hemodynamics. Journal of clinical ultrasound, 36(3), 134-140.

36. Tana, C., Tana, M., Rossi, S., et al. (2016). Hepatic artery resistive index (HARI) and non-alcoholic fatty liver disease (NAFLD) fibrosis score in NAFLD patients: cut-off suggestive of nonalcoholic steatohepatitis (NASH) evolution. Journal of ultrasound, 19(3), 183-189.

37. Ulusan, S., Yakar, T., and Koc, Z. (2011). Evaluation of portal venous velocity with Doppler ultrasound in patients with nonalcoholic fatty liver disease. Korean journal of radiology, 12(4), 450455.

38. Wajchenberg, B. L. (2000). Subcutaneous and visceral adipose tissue: their relation to the metabolic syndrome. Endocrine reviews, 21(6), 697738.

39. Seifalian, A. M., El-Desoky, A., \& Davidson, B. R. (2001). Hepatic indocyanine green uptake and excretion in a rabbit model of steatosis. European surgical research, 33(3), 193-201.

40. Baikpour, M., Ozturk, A., Dhyani, M., et al. (2020). Portal venous pulsatility index: a novel biomarker for diagnosis of high-risk nonalcoholic fatty liver disease. American Journal of Roentgenology, 214(4), 786-791.

41. McNaughton, D. A., and Abu-Yousef, M. M. (2011). Doppler US of the liver made simple. Radiographics, 31(1), 161-188.

42. RangankaR, V. P., Bhole, P. R., Gosavi, S. S., et al. (2018). Ultrasound of Hepatic Vessels in Fatty Liver Disease.

43. Topal, N. B., Orcan, S., Sığırlı, D., et al. (2015). Effects of fat accumulation in the liver on hemodynamic variables assessed by Doppler ultrasonography. Journal of Clinical Ultrasound, 43(1), 26-33.

44. Iliopoulos, P., Vlychou, M., Margaritis, V., et al. (2007). Gray and color Doppler ultrasonography in differentiation between chronic viral hepatitis and compensated early stage cirrhosis. Journal of Gastrointestinal and Liver Diseases, 16(3), 279.

45. PIERCE, M. E., and Sewell, R. (1990). Identification of hepatic cirrhosis by duplex Doppler ultrasound value of the hepatic artery resistive index. Australasian radiology, 34(4), 331-333.

46. Colli, A., Cocciolo, M., Mumoli, N., et al. (1998). Hepatic artery resistance in alcoholic liver disease. Hepatology, 28(5), 1182-1186.

47. Hizli, Ş., KOÇYİĞiT, A., Arslan, N., et al. (2010). The role of ultrasonographic hepatic artery resistive index in the diagnosis of insulin resistance in obese children with non-alcoholic fatty liver disease. Turkish Journal of Medical Sciences, 40(3), 335-342.

48. Mohammadi, A., Ghasemi-rad, M., Zahedi, H., et al. (2011). Effect of severity of steatosis as assessed ultrasonographically on hepatic vascular indices in non-alcoholic fatty liver disease. Medical ultrasonography, 13(3), 200-206.

49. Tana, C., Schiavone, C., Ticinesi, A., et al. (2018). Hepatic artery resistive index as surrogate marker for fibrosis progression in NAFLD patients: A clinical perspective. International journal of immunopathology and pharmacology, 32, 2058738418781373.

50. Mohammadinia, A. R., Bakhtavar, K., EbrahimiDaryani, et al. (2010). Correlation of hepatic vein Doppler waveform and hepatic artery resistance index with the severity of nonalcoholic fatty liver disease. Journal of Clinical Ultrasound, 38(7), 346352. 